\title{
Microstates in Language-Related Brain Potential Maps Show Noun-Verb Differences
}

\author{
Thomas Koenig And Dietrich Lehmann
}

\author{
EEG-EP Mapping Laboratory, Department of Neurology, University Hospital, \\ CH-8091 Zurich, and The KEY Institute for Brain-Mind Research, University Hospital of \\ Psychiatry, CH-8029 Zurich, Switzerland
}

\begin{abstract}
Brain processing of grammatical word class was studied analyzing event-related potential (ERP) brain fields. Normal subjects observed a randomized sequence of single German nouns and verbs on a computer screen, while 20-channel ERP field map series were recorded separately for both word classes. Spatial microstate analysis was applied, based on the observation that series of ERP maps consist of epochs of quasi-stable map landscapes and based on the rationale that different map landscapes must have been generated by different neural generators and thus suggest different brain functions. Space-oriented segmentation of the mean map series identified nine successive, different functional microstates, i.e., steps of brain information processing characterized by quasi-stable map landscapes. In the microstate from 116 to $172 \mathrm{msec}$, noun-related maps differed significantly from verb-related maps along the left-right axis. The results indicate that different neural populations represent different grammatical word classes in language processing, in agreement with clinical observations. This word class differentiation as revealed by the spatialtemporal organization of neural activity occurred at a time after word input compatible with speed of reading. (๑) 1996 Academic Press, Inc.
\end{abstract}

\section{INTRODUCTION}

The assessment of selective language impairments of aphasic patients has advanced the knowledge about the organization of language in the brain. Experience with patients presenting with dissociations between semantic categories suggests that one of the dimensions of lexical organization of the brain is semantic. Semantic categories can be of the type abstract vs. concrete (Warrington \& Shallice, 1984), living things and food vs. inanimate objects (Warrington \& McCarthy, 1983, 1987; Basso, Capitani, \& Laiacona, 1988; Hillis \& Caramazza, 1991), animals (Hillis \& Caramazza, 1991), countries

The study has been supported by a grant from the Swiss National Science Foundation (3130274.90).

Address reprint requests to Dr. Thomas Koenig, The KEY Institute for Brain-Mind Research, University Hospital of Psychiatry, CH-8029 Zurich, Switzerland. 
(McKenna \& Warrington, 1978), fruits and vegetables (Hart, Berndt, \& Caramazza, 1985), and others. Additional studies reported selective impairment of nouns or verbs, implying that grammatical class seems to be another important dimension of lexical organization (Miceli, Silveri, Villa, Caramazza, 1984; Miceli, Silveri, Nocentini, \& Caramazza, 1988; McCarthy \& Warrington, 1985; Zingeser \& Berndt, 1988, 1990; Caramazza \& Hillis, 1991).

The measurement of event-related potentials (ERPs) has proven to be a useful method in investigations of brain processing of language. ERPs were shown to deviate if the subject's semantic expectancies were violated by incongruous sentence endings (e.g., Kutas \& Hillyard, 1980; Fischler, Bloom, Childers, Arroyo, \& Perry, 1984; Besson, Kutas, \& Van Petten, 1992; Curran, Tucker, Kutas, \& Posner, 1993; Karniski, Vanderploeg, \& Lease, 1993; see Kutas \& Van Petten, 1988, for a review). Aphasic patients do not show the same deviations (Brandeis \& Lehmann, 1994). Other ERP deviations were observed if the subject's grammatical expectancies were violated (Neville, Nicol, Barss, Forster, \& Garrett, 1991; Van Petten \& Kutas, 1991; Rösler, Pütz, Friderici, \& Hahne, 1993). Chapman, McCrary, Chapman, and Martin, (1980) found that ERP responses to single words differed depending on the subjects' rating of the stimuli on Osgood's (1952) semantic differential scales.

The noun-verb distinction is considered in linguistics as a fundamental property of human language, and it seems to appear in all languages with only one notable exception (page 4 in Greenberg, 1968). There were only few adequate attempts to describe this difference in terms of spatial and temporal properties of the brain's undisturbed language processing in healthy subjects. Teyler, Roemer, and Harrison (1973) showed that ERPs are altered depending on whether subjects kept in mind a noun or a verb during a click stimulation. Brown and Lehmann (1979) found that noun and verb meaning of auditorily presented homophone words involve different neural generators similarly for German, English, and suggested language presentation. However, in both studies, subjects were aware of the word meaning before the actual presentation, so that the observed ERP differences cannot distinguish between an initially altered brain state and differences in processing of the incoming information. Samar and Berent (1986) reported an ERP principal component factor that significantly accounted for grammatical class. In their study, a lexical decision task was used so that visually presented nouns or verbs or nonwords were embedded in a grammatical context by a prime stimulus word.

Differences in brain information processing correspond to different spatial patterns of neural activity in the brain. These differences in neural activity can be detected as differences of scalp electric field topography. A change in this topography over time indicates a transition from one functional process to another one because different scalp field topographies must have been 
produced by the activity of nonidentical neural populations (see Fender, 1987) and because it is reasonable to assume that the activities of different neural ensembles implement different functions.

Changes in brain electric field topography over time are discontinuous and tend to occur in steps. These step-wise changes of topography are interspersed between longer time epochs of quasi-stable field topographies in the subsecond range, the functional microstates (Lehmann \& Skrandies, 1984; Brandeis \& Lehmann, 1986, 1989; Lehmann, 1987). The microstates are suggested to be the basic elements of stepwise processing of information in the brain.

The aim of the present study was to investigate, in normal subjects, whether the processing of randomly presented single nouns and verbs involves different neural structures as suggested by the patient observations, using a task that does not invite grammatical processing. If, during the processing of presented stimuli, one of the ERP microstates was altered consistently by the word's grammatical class and thus identified a difference in the brain's language processing steps, it would indicate that different structures are active for the two word classes, and it would establish the time after input during which the distinction is made. Temporal segmentation of the ERP map series into microstates was applied, and the microstates were compared between noun and verb condition in order to describe the time course of the brain's language processing steps and their functional significance.

\section{METHODS}

Subjects. The subjects were 13 female and 4 male, paid volunteers (mean age 26 years, range 19-51). The first language of all subjects was Swiss German or High German; there were no drug or medication users. Fifteen subjects were right-handed and eleven of them had no left-handed relatives. Vision was normal or corrected to normal. All subjects gave their informed consent to the procedure.

Stimuli. The word stimuli were taken from an index of word frequency of German written language (Rosengren, 1977). Nouns and verbs with two syllables were chosen; they did not differ in average word frequency and number of letters (average logarithm of frequency $=$ 6.23, average number of letters 6.40). Words that can be used both as nouns and verbs (e.g., "RENNEN" = "RUN") were excluded. The final stimuli were 63 nouns and 63 verbs; they were randomly divided into three blocks of 42 words each. Each block was used four times, making a total of twelve blocks of stimuli whose sequence was randomized over subjects. Within each block of 42 word stimuli, the words were presented in random sequence. All words were presented in capital letters.

Presentation of stimuli. The subjects were seated in a darkened, electrically and soundshielded chamber and viewed a computer monitor at a distance of $125 \mathrm{~cm}$. All instructions and stimuli were displayed in black letters on light grey. A five-letter word covered $5.3 \times$ $1.3 \mathrm{~cm}$, i.e., a visual angle of $2.4 \times 0.6$ degrees. The subject was instructed to read each word silently and to repeat orally the last word whenever a question mark appeared. These question marks followed a word stimulus after $1800 \mathrm{msec}$ with a $10 \%$ random probability. Within each block, the word stimuli were presented for $450 \mathrm{msec}$ at intervals of $1800 \mathrm{msec} ; 42$ stimuli formed a block; pauses of 1.5 min separated the blocks. 

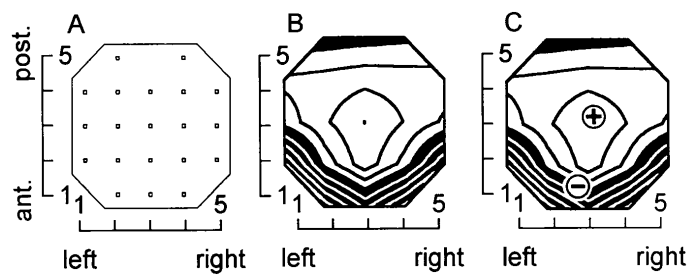

FIG. 1. Spatial feature extraction. (A) Schematic of the electrode array on the scalp; head seen from above, left ear left. Electrode $\mathrm{Cz}$ is at the center. Tick marks at the coordinate axes indicate electrode numbers, in the anterior-posterior direction (vertical axis) and in the leftright direction (horizontal axis). (B) Example of a momentary potential distribution map. Scalp electric potentials were measured at the locations shown in A, linearly interpolated, and displayed as isopotential contour line maps using steps of $1.0 \mu \mathrm{V}$. White is positive, black negative, referred to the mean of all values. (C) Centroids (centers of gravity) of the positive and negative map area computed for spatial feature extraction. The configuration of the map is described by the anterior-posterior and left-right coordinates of the positive and negative area centroids.

Data acquisition. Electrodes were attached at scalp positions Fp1/2, F7/8, F3/4, Fz, T3/4, $\mathrm{C} 3 / 4, \mathrm{Cz}, \mathrm{T} 5 / 6, \mathrm{P} 3 / 4, \mathrm{Pz}, \mathrm{O} 1 / 2$, and $\mathrm{Oz}$ of the international 10-20 system (Fig. 1A).

One electrode at the outer left canthus recorded eye movements. Impedances were below $5 \mathrm{kOhms}$. Fp1 served as recording reference; the ground electrode was placed at Fpz. The 20-channel data were amplified $(0.1-30 \mathrm{~Hz}$ bandpass), digitized (250 s/sec), and averaged on-line using a Biologic Brain Atlas System. For additional off-line digitizing and analysis, the raw EEG and stimulus signals of the last 13 subjects were recorded continuously on 21channel FM analog tape. Averaging covered $1024 \mathrm{msec}$ after stimulus onset. Automatic artifact rejection was $60 \mu \mathrm{V}$ for eye movements and $150 \mu \mathrm{V}$ for ERP channels. Separate averages were obtained for verbs and nouns during each of the 12 stimulus blocks. In 7 of the total 204 presented blocks, the subjects failed to repeat the last word after the question mark; these data were excluded from further analysis.

All data were recomputed off-line into average reference 20-channel ERP map series. For each subject, one mean ERP map series for all noun stimuli and one for all verb stimuli was computed over all stimulus blocks, weighted by the number of sweeps available for the respective word class. The resulting 34 mean 20-channel ERP map series were visually inspected for artifacts; 4 of the constituting $34 \times 20=680$ waveshapes were contaminated and were linearly interpolated between the neighbor electrodes.

Analysis. For the numerical assessment of the map topography, the locations of the centroid of the positive and the negative areas of each map were computed (Wackermann, Lehmann, Michel, \& Strik, 1993). The positive and negative centroids are the location points of gravity of the positive and negative areas of the (spatial offset-corrected) potential distribution maps. The centroids describe the spatial configuration of a momentary map by four spatial coordinate values, independent of map strength. An example is shown in Fig. 1C.

The four coordinate values of the centroid locations of each map were averaged separately across the 17 subjects and the two conditions. These grand mean centroids were used for microstate segmentation.

Segmentation of the ERP map series into microstates. The procedure for segmenting the map series into successive periods of quasi-stable field configuration or microstates (Lehmann \& Skrandies, 1984; Brandeis \& Lehmann, 1986; Lehmann, 1987) is briefly reviewed in the following.

A microstate is started by setting one spatial window of a certain size around the positive 
1
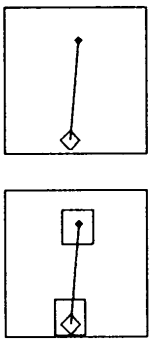

2
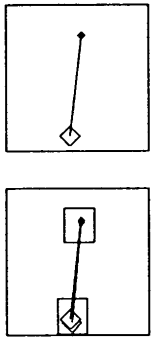

3
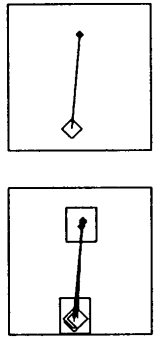

4
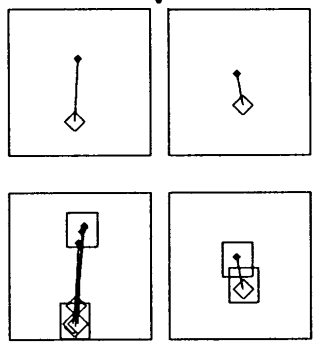

6
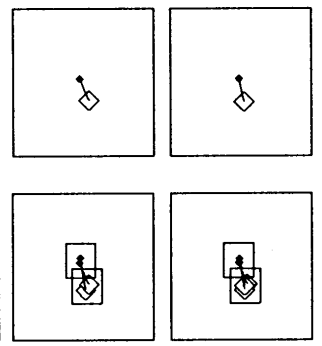

FIG. 2. Example of segmentation into microstates using centroids as descriptors of the map landscapes evoked by nouns and verbs (the grand mean centroids from 140 to $188 \mathrm{msec}$ are illustrated in steps of $8 \mathrm{msec}$ ). Filled dots show the positive, open diamonds the negative centroids; each centroid pair is connected by a line for easier viewing. Each time frame is drawn twice; the top graph shows the momentary locations of the centroids; the bottom graph shows, in addition, the location of all centroids accumulated so far within the microstate, surrounded by their spatial windows (squares). The windows set around the centroids of frame 1 also accommodated those of frames 2, 3, and 4 after some translation, but the centroids of frame 5 lay outside. Thus, a new segment was started with frame 5; it continued through frames 6 and 7 .

centroid and one around the negative centroid of the first map (frame 1 of Fig. 2). The two centroids of the next map are projected into this graph (frame 2 of Fig. 2). By translating the window that surrounds the positive centroid of map 1 it was attempted to accommodate the new positive centroid within the window while still including the previous one; the same is attempted for the negative centroid and its window. If accommodation is possible, the map belongs to the current microstate and the following map is considered for inclusion (frames 3 and 4 in Fig. 2). If it is not possible to accommodate the next centroids within their appropriate windows unless a previous one was left out, a microstate border is accepted and a new microstate is started (frame 5 in Fig. 2).

Obviously, microstate duration must increase with increasing window size-a wide open window will find no microstate borders. In order to obtain a general measure of map landscape stability across time, the segmentation was applied repeatedly using all window sizes from zero to 1.0 electrode distance (ED) in steps of $0.01 \mathrm{ED}$. The obtained microstate borders were summed for each moment in time, resulting in a microstate border probability as a function of time. This yielded the probability of encountering a microstate border across window sizes as a function of time. The curve of microstate border probability was digitally low-passed at $30 \mathrm{~Hz}$ (FIR filter). The final curve (Fig. 3) showed various clear peaks, indicating that there are preferred moments in time for changes of map topography.

Segmentation must equally satisfy two opposing goals, the assignment of a maximal number of maps to microstates (stability criterion) and the detection of a maximal number of microstates (discrimination criterion). Stability was defined as total number of time points with a microstate border probability between two successive maps below a certain level. Discrimination was defined as total number of epochs between peaks of the microstate border probability functions that were above a certain level. The optimal level of microstate border probability was defined as that level where the product "stability measure multiplied by discrimination measure" had its absolute maximum. This optimal level is indicated by the horizontal line in Fig. 3. The latencies of the peaks above optimal level were accepted as microstate borders. 
Microstate border probability

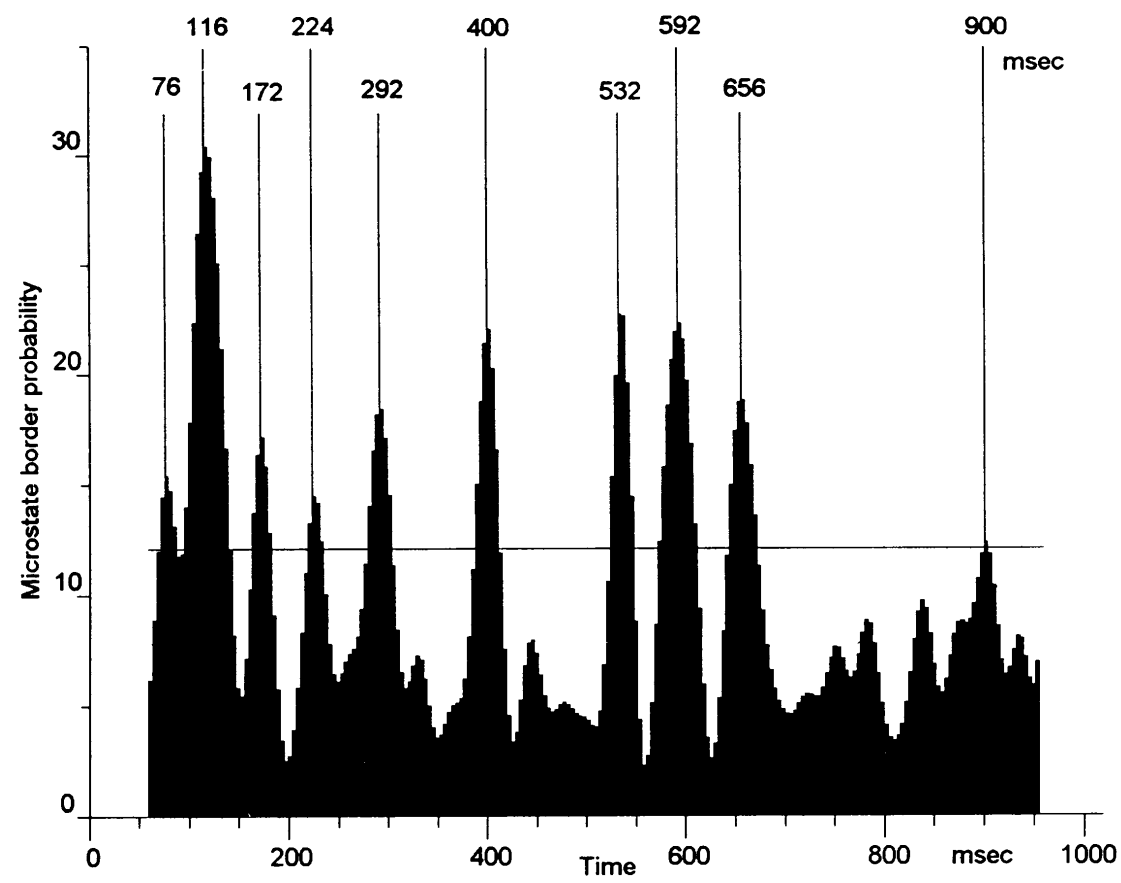

FIG. 3. Identification of the microstates. The probability of microstate borders (vertical) as a function of time (horizontal) is shown as black area. The peaks of this microstate border probability graph indicate the preferential time instants of map landscape change. The optimal level of landscape change accepted for the identification of the final microstates (see text) is drawn in as a horizontal line.

Statistics. A split-half replication approach was used. The subjects were partitioned into two groups in the sequence of the recording dates. The location of the centroids of either condition were separately averaged within each microstate in each of the first nine subjects and compared with two-tailed paired $t$ tests (four $t$ values for each microstate) between conditions. Cases with double-ended $p$ smaller than 0.10 were accepted as interesting hypotheses; the cut-off $p$ level was set to $10 \%$ in order to minimize the number of confirmatory tests without excluding promising candidate hypotheses (Type II errors). The hypotheses generated with the first nine subjects were tested in the second eight subjects using confirmatory statistics.

Additional analyses. It might be hypothesized that differences between noun and verb condition emerge not from the difference along the grammatical dimension but from systematic differences in some semantic category. In order to clarify this question, 12 new volunteers (mean age 40.4, range 23-63) rated all stimuli on Osgood's (1952) semantic scales. Each stimulus word was presented on a computer screen and the subject rated it on the scale "evaluation" ("negative $<->$ positive") or "activity" ("passive $<->$ active") or "strength" ("weak $<->$ strong") by assigning it to an integer value between 1 and 5 . The word sequence was randomized, the scale sequence was balanced across subjects, and the scales were reversed in half of the cases. The program ended when all stimulus words had been rated on the three scales. There was no significant difference between nouns and verbs on the scales "evalua- 

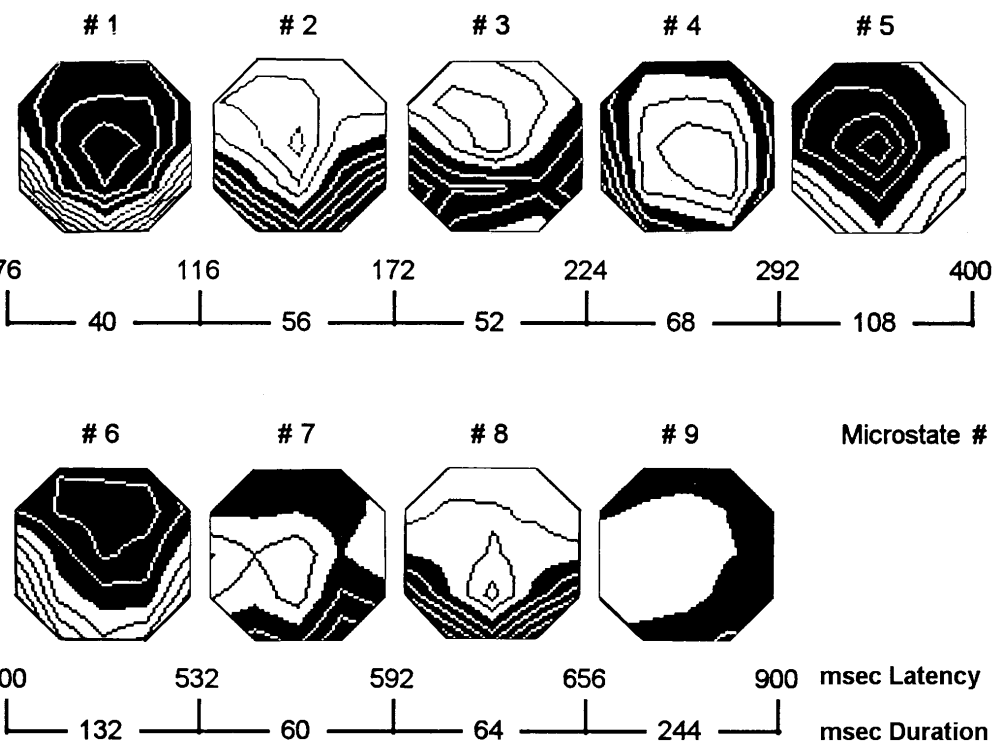

FIG. 4. Grand mean potential distribution maps of the nine microstates with their durations and latencies. Head seen from above, left ear left, white positive, black negative vs. average reference. Linear interpolation between electrode positions, isopotential contour lines in steps of $1.5 \mu \mathrm{V}$.

tion" and "strength," but nouns were significantly lower than verbs $(t=3.79$, df $=11$, $p=.003)$ on the scale "activity.",

Because of this result, two additional off-line ERP analyses were carried out using the digitized, continuous recordings of the last 13 subjects. Analysis (1) compared ERPs from a subset of nouns against a subset of verbs, where nouns and verbs were matched for word frequency and "activity", rating, thus excluding differences on the "activity" scale. Analysis (2) used ERPs from two subsets of the word stimuli that were maximally different on the "activity" scale and did not differ in word frequency and number of nouns and verbs. This analysis was expected to maximize the effect of the difference in the "activity" ratings and exclude any grammatical difference. All data were treated as in the main analysis. Statistical comparisons were computed for the microstate where differences between nouns and verbs had been encountered in the previously described main analysis.

\section{RESULTS}

The segmentation of the data identified nine microstates with durations between 40 and $244 \mathrm{msec}$ as shown in Fig. 3.

Figure 4 displays the average potential distribution maps of these microstates and their durations. The microstate at 76-116 msec showed the typical configuration of the "P100" component of visual evoked potentials with an occipital positivity.

In the first group of nine subjects, the differences of the four location parameters of the centroids (positive and negative centroid, left-right and 

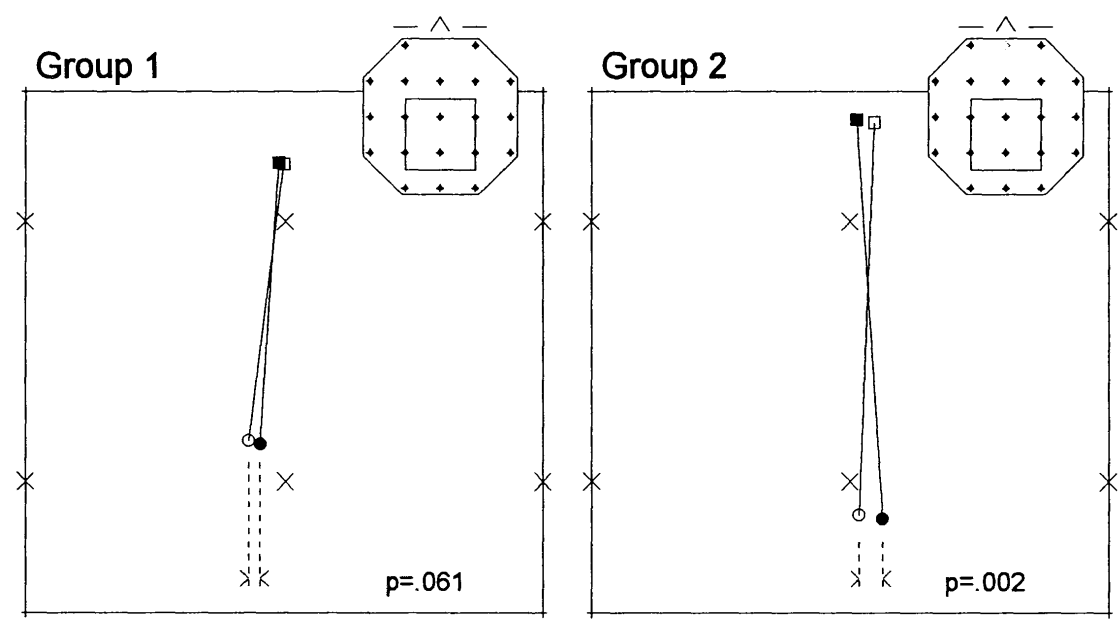

FIG. 5. Noun-verb difference in the second microstate at 116-172 msec poststimulus. The mean locations (across subjects) of the centroids of the maps of this microstate are shown for the subjects of group 1 (left) and group 2 (right). Solid centroids, verb data; open centroids, noun data. Anterior centroids (squares) are positive; posterior centroids (circles) are negative. Significancies of the location differences are indicated (details see text). Electrode positions are marked by x's. (Inset) Position of the enlarged area on the head.

anterior-posterior axis) between the noun and verb condition were tested for each microstate, using paired $t$ tests. Two of the 36 tests showed significance levels (double-ended, $\mathrm{df}=8$ ) at $p<.10$. In the second microstate $(116-172 \mathrm{msec})$, the average negative centroid had a location more to the left for nouns than for verbs $(t=2.18, p=.061)$, and in the third microstate $(172-224 \mathrm{msec})$, the average positive centroid had a location more to the left for nouns than for verbs $(t=3.25, p=.012)$.

These two results were accepted as hypotheses and were tested with the second group of eight subjects. The left-right difference of the negative centroid in the second microstate $(116-172 \mathrm{msec})$ was confirmed at a significance level of $p=.002$ (single ended, $t=4.30, \mathrm{df}=7$ ). The difference in the third microstate $(172-224 \mathrm{msec})$ was not confirmed. Combining the data of all subjects, the left-right difference of the negative centroid in the second microstate was significant at $p=.001$ (double ended, $t=4.12$, df $=16$ ) after Bonferroni correction for $9 \times 4$ tests $=.036$.

Figure 5 illustrates the mean centroid locations over subjects of the nounand verb-evoked maps of the second microstate, for the first nine and the second eight subjects.

The additional analyses indicated that the difference between the two stimulus conditions was clearly due to the difference in grammatical class and not to the difference on Osgood's (1952) 'activity"' scale: In analysis (1), which excluded any differences in "activity" between nouns and verbs, the 
difference in the second microstate persisted (single-ended $t$ test, $t=2.13$, $\mathrm{df}=12, p=.028$ ). Analysis (2) focused on the difference in rated "activity" and excluded any difference in grammatical class; there was no significant difference in the second microstate between "active" and "passive" words (single-ended $t$ test, $t=0.105$, df $=12, p=.46$ ).

\section{DISCUSSION}

The results showed that in a simple, single word reading paradigm, the brain processes nouns and verbs through different neural populations. This is concluded from the observation that during a defined period of time (during the microstate 116-172 msec poststimulus), the spatial configuration of the evoked brain electric field differed between nouns and verbs, thus implying that the combination of active sources within the brain was nonidentical, i.e., that it differed in location and/or orientation (Fender, 1987).

The present finding is in good agreement with reports on aphasic patients who showed selective impairments of nouns or verbs (Miceli et al., 1984, 1988; McCarthy \& Warrington, 1985; Zingeser \& Berndt, 1988, 1990; Caramazza \& Hillis, 1991). It is important that the subjects had only been instructed to read and, if prompted, to repeat the presented word. The subjects were not informed about the objective of the study, i.e., the possible differences in brain fields evoked by nouns vs. verbs.

The analysis used space-oriented methods that permited discrimination between the activity of different neural ensembles, independent of their strength and latency. The utilized adaptive segmentation strategy allows one to describe the data as a sequence of brain microstates of different durations and landscapes. The segmentation in the present analysis employed a datadriven decision level instead of the preselected, fixed identity criterion of the original segmentation methodology (Lehmann \& Skrandies, 1984; Lehmann, 1987; Brandeis \& Lehmann, 1989). The segmentation procedure may use spatial data reduction (as in the present approach) or, at the other extreme, directly treat the original values at all locations (see, e.g., Global Map Dissimilarity in Lehmann, 1987, p. 334). In the present analysis, the map topographies were assessed by a feature extraction that resulted in the centroid descriptors. It has been shown that the latencies of the microstates are closely similar if the maps are compared directly by correlating their voltage distributions (full topographic information applying Global Map Dissimilarity) instead of the extracted spatial features and even if the latencies of the distribution-independent Global Field Power minima are used as in Michel and Lehmann (1993).

Using a denser electrode array might have rendered the recordings still more sensitive to details of the spatial map configuration. However, the presently measured microstate topographies at comparable latencies resembled those of other studies that used higher density electrode arrays. The micro- 
state present from 76 to $116 \mathrm{msec}$ (P100) had bioccipital positivities that corresponded well with the bioccipital positive extrema observed with 42electrode potential maps (Michel \& Lehmann, 1993) and with 64-electrode current source density maps (Curran et al., 1993). Similarly, the topography of the microstate from 116 to $172 \mathrm{msec}$ with its bioccipital negative peaks is comparable (although slightly earlier) to that of the so-called N1/P2 complex of Curran et al. (1993, Fig. 5).

The high temporal resolution offered by brain electric data reveals the development of language-related activity over time. Using the spatial segmentation procedure, a functional microstate of the brain activity was identified between 116 and $172 \mathrm{~ms}$ postpresentation with a topography that was consistently biased by the grammatical class of the incoming word, i.e., noun vs. verb, indicative of different active neural generators. No such effect was observed in the previous and in the following microstate. This suggests a well-defined time course of the brain's language processing, where one step, defined by the geometry of the activated neural populations and by its latency after input reflects the noun-verb distinction.

The observation of the grammatical class-sensitive microstate at 116-172 msec after input indicates that language processing in the brain begins much earlier than the repeatedly studied N400 component evoked by semantic incongruity (for references see Introduction).

Some previous ERP studies using noun and verb stimuli had also shown early differences (Teyler et al., 1973; Brown \& Lehmann, 1979; Brown, Marsh, \& Smith, 1979). Only limited comparisons with the present study are possible as in those experiments, the subjects were aware of the meaning of the stimuli before they were presented, and stimulation was auditory. However, one significant result of the Brown and Lehmann data was a leftright difference of the maps' negative extreme locations between 33 and 177 msec poststimulus (Lehmann \& Skrandies, 1984) in the same sense as in the present study.

Samar and Berent (1986) used a syntactic priming paradigm with a lexical decision task were visually presented prime words created a grammatical expectancy and were followed by a noun or verb target word or a nontarget nonword. The authors found an ERP principal component factor accounting significantly for correct versus incorrect priming, independent of the word's grammatical class; it was largest at $140 \mathrm{msec}$ poststimulus. A different factor, accounting significantly for grammatical class independent of the word's correct grammatical usage, peaked only $80 \mathrm{msec}$ later. These results are in some contradiction to those of the present study, where effects of grammatical class were found during the microstate at 116-172 msec poststimulus. It remains to be seen whether this difference resulted from the different experimental paradigm or from different analysis strategies (principal component mastoid-referenced waveshape analysis versus space-oriented microstate analysis). 
Some other studies reported language-related ERP differences in a comparable early poststimulus time range. In a speeded category decision task, Boddy and Weinberg (1981) found priming effects in visually evoked ERPs within a latency range of 130-212 msec. Heine, Roth, Koenig, and Knye (1993) studied ERPs evoked by subliminally presented words yielding to conscious identification only after several repetitions of the presentation. They observed a negative ERP component peaking at about $140 \mathrm{msec}$ that was influenced by auditory priming and subjective word frequency, although subjects could not consciously reproduce the stimulus words. Neville and collaborators (1991) observed a negativity around $125 \mathrm{msec}$ that was enhanced by violations of the expected phrase structure generated by grammatical information. In an auditory paradigm, Wetzel and Molfese (1992) identified a component with an early peak at $144 \mathrm{msec}$ that was sensitive to presuppositional information contained in sentences. Curran and collaborators (1993) observed an ERP component at 136-184 msec that was different for congruent and incongruent sentence endings; the authors noted that the same component was selectively left-lateralized if semantic processing was required. Reading is comfortable at a speed of about five words per second (Fischler \& Bloom, 1980) and semantic priming effects occur if only 250 msec are available to process the prime (Neely, 1977). In agreement with the present results, these observations suggest that at least some of the semantic processing of incoming information occurs within the first $200 \mathrm{msec}$. The quoted reports imply that this processing need not always lead to conscious experiences.

The current results indicate that the neural organization of recognition of grammatical class is similar across individuals. It is encouraging to find common features of language processing in normal subjects as it suggests that language deficits can be assigned to specific steps of brain information processing. Deficits in language processing might be associated with changes in the geometry of neural activity (Brandeis \& Lehmann, 1994) as well as with altered time course (Harbin, Marsh, \& Harvey, 1984), demonstrating that both spatial and temporal features of brain electric data are needed to describe adequately normal and altered language processing.

Language processing involves multiple essential areas of the brain as well as widely dispersed neurons, as has been shown in intraoperative electric stimulations of brain tissue (Ojemann, 1991). Thus, it is to be assumed that the stimuli in the present study activated large multifocal neural systems (Mesulam, 1990) of which at least partially different subsets were engaged for nouns and verbs. The scalp topography of the brain's electric field reflects the sequence of activity of the extended neural areas. The numerical description of the topographies showed a statistically confirmed difference between the neural populations activated during noun versus verb processing. This difference was numerically small because it juxtaposed the center-of-gravity locations of basically related, widespread neural activity patterns. The crucial 
conclusion is that the involved neural populations were nonidentical in a consistent manner over subjects.

It is not possible to unequivocally assign anatomical structures to different brain electric microstates because the inverse problem of the field equations for an unknown number of generators is nonunique (Fender, 1987). Even though the field topographies might be focalized by data transformations such as spatial high pass filtering (e.g., current source density computations), the putative sources cannot be assumed to be located perpendicular below the scalp distribution extrema (e.g., the "paradoxical lateralization" of the visual P100 (Barrett, Blumhardt, Halliday, Halliday, \& Kriss, 1976). However, the location of the mean of the two centroid locations of a potential map is a simple estimate of the surface-projected location of an assumed single electric source that explains the field; this location of the observed noun mean fields was more to the left than that of the verb mean fields (double-ended $p=.020, t=2.95$, df $=16$ ).

Studies using positron emission tomography have described brain regions that are activated in language-related tasks compared with a baseline condition (Posner, Petersen, Fox, \& Raichle, 1988; Wise, Chollet, Hadar, Friston, Hoffner, \& Frackowiak, 1991; Mazoyer, Tzourio, Frak, Syrota, Murayama, Levrier, Salamon, Dehaene, Cohen, \& Mehler, 1993), but they cannot provide information about the time course of the activation patterns. As these patterns change rapidly and drastically in the subsecond time range, time information appears to be important. For a deeper understanding of normal and pathological language processing in the human brain, the application of both methods will be useful as they provide complementary information.

\section{REFERENCES}

Barrett, G., Blumhardt, L., Halliday, A. M., Halliday, E., \& Kriss, A. 1976. A paradox in the lateralization of the visual evoked response. Nature, 261, 253-255.

Basso, A., Capitani, E., \& Laiacona, M. 1988. Progressive language impairment without dementia: A case with isolated category specific semantic defect. Journal of Neurology, Neurosurgery, and Psychiatry, 51, 1201-1207.

Besson, M., Kutas, M., \& Van Petten, C. 1992. An event-related potential (ERP) analysis of semantic congruity and repetition effects in sentences. Journal of Cognitive Neuroscience, 4, 132-149.

Boddy, J., \& Weinberg, H. 1981. Brain potentials, perceptual mechanisms and semantic categorization. Biological Psychology, 12, 43-62.

Brandeis, D., \& Lehmann, D. 1986. Event-related potentials of the brain and cognitive processes: Approaches and applications. Neuropsychologia, 24, 151-168.

Brandeis, D., \& Lehmann, D. 1989. Segments of event-related potential map series reveal landscape changes with visual attention and subjective contour. Electroencephalography and Clinical Neurophysiology, 76, 507-519.

Brandeis, D., \& Lehmann, D. 1994. ERP mapping: A tool for assessing language disorders? In H. J. Heinze, T. F. Münte, \& G. R. Mangun (Eds.), Cognitive Electrophysiology. Boston: Birkhäuser.

Brown, W. S., \& Lehmann, D. 1979. Verb and noun meaning of homophone words activate different cortical generators: A topographical study of evoked potential fields. Experimental Brain Research, Suppl. 2, 159-168. 
Brown, W. S., Marsh, J. T., \& Smith, J. C. 1979. Principal component analysis of ERP differences related to the meaning of an ambiguous word. Electroencephalography and Clinical Neurophysiology, 46, 709-714.

Caramazza, A., \& Hillis, A. E. 1991. Lexical organization of nouns and verbs in the brain. Nature, 349, 788-789.

Chapman, R. M., McCrary, J. W., Chapman, J. A., \& Martin, J. K. 1980. Behavioral and neural analysis of connotative meaning: Word classes and rating scales. Brain and Language, 11, 319-339.

Curran, T., Tucker, D. M., Kutas, M., \& Posner, M. I. 1993. Topography of the N400: Brain electrical activity reflecting semantic expectancy. Electroencephalography and Clinical Neurophysiology, 46, 709-714.

Fender, D. H. 1987. Source localization of brain electrical activity. In. A. S. Gevins \& A. Remond (Eds.), Methods of analysis of brain electrical and magnetic signals. Handbook of electroencephalography and clinical neuropsychology. Amsterdam: Elsevier. Vol. 1.

Fischler, I., \& Bloom, P. A. 1980. Rapid processing of the meaning of sentences. Memory and Cognition, 8, 216-225.

Fischler, I., Bloom, P. A., Childers, D. G., Arroyo, A. A., \& Perry, N. W. 1984. Brain potentials during sentence verification: Late negativity and long-term memory strength. Neuropsychologia, 22, 559-568.

Greenberg, J. H. 1968. Universals of language. Cambridge, MA: MIT Press. 2nd ed.

Harbin, T. J. Marsh, G. R., \& Harvey, M. T. 1984. Differences in the late components of the event-related potential due to age and to semantic and non-semantic tasks. Electroencephalography and Clinical Neurophysiology, 59, 489-496.

Hart, J., Berndt, R. S., \& Caramazza, A. 1985. Category-specific naming deficit following cerebral infarction. Nature, 316, 439-440.

Heine, A., Roth, N., Koenig, T., \& Knye, M., 1993. Effects of auditory priming and word frequency upon ERP and task performance during word recognition. In H. J. Heinze, T. F. Münte, \& G. R. Mangun (Eds.), New developments in event-related potentials. Boston: Birkhäuser.

Hillis, A. E., \& Caramazza, A. 1991. Category-specific naming and comprehension impairment: A double dissociation. Brain, 114, 2081-2094.

Karniski, W., Vanderploeg, R., \& Lease, L. 1993. 'Virtual N400'” and slow wave topography to auditory sentence incongruence. Brain and Language, 44, 58-79.

Kutas, M., \& Hillyard, S. A. 1980. Reading senseless sentences: Brain potentials reflect semantic incongruity. Science, 207, 203-205.

Kutas, M., \& Van Petten, C. 1988. Event-related brain potential studies of language. In J. R. Jennings \& M. G. H. Coles (Eds.), Advances in psychophysiology. Greenwich: JAI Press.

Lehmann, D. 1987. Principles of spatial analysis. In A. S. Gevins \& A. Remond (Eds.), Methods of analysis of brain electrical and magnetic signals. Handbook of electroencephalography and clinical neurophysiology. Amsterdam: Elsevier. Vol. 1.

Lehmann, D., \& Skrandies, W. 1984. Spatial analysis of evoked potentials in man-A review. Progress in Neurobiology, 23, 227-250.

Mazoyer, B. M., Tzourio, N., Frak, V., Syrota, A., Murayama, N., Levrier, O., Salamon, G., Dehaene, S., Cohen, L., \& Mehler, J. 1993. The cortical representation of speech. Journal of Cognitive Neuroscience, 5, 467-479.

McCarthy, R., \& Warrington, E. K. 1985. Category specificity in an agrammatic patient: The relative impairment of verb retrieval and comprehension. Neuropsychologia, 23, 709-727.

McKenna, P., \& Warrington, E. K. 1978. Category-specific naming preservation: A single case study. Journal of Neurology, Neurosurgery and Psychiatry, 41, 571-574.

Mesulam, M. 1990. Large-scale neurocognitive networks and distributed processing for attention, language and memory. Annals of Neurology, 28, 597-613.

Miceli, G., Silveri, M. C., Villa, G., \& Caramazza, A. 1984. On the basis for the agrammatic's difficulty in producing main verbs. Cortex, 20, 207-220. 
Miceli, G., Silveri, M. C., Nocentini, U., \& Caramazza, A. 1988. Patterns of dissociation in comprehension and production of nouns and verbs. Aphasiology, 2, 351-358.

Michel, C. M., \& Lehmann, D. 1993. Single doses of piracetam affect 42-channel event-related potential microstate maps in a cognitive paradigm. Neuropsychobiology, 28, 212-221.

Neely, J. H. 1977. Semantic priming and retrieval from semantic memory: Roles of inhibitionless spreading activation and limited-capacity attention. Journal of Experimental Psychology: General, 106, 226-254.

Neville, H., Nicol, J. L., Barss, A., Forster, K. I., \& Garrett, M. F. 1991. Syntactically based sentence processing classes: Evidence from event-related brain potentials. Journal of Cognitive Neuroscience, 3, 151-165.

Ojemann, G. A. 1991. Cortical organization of language. The Journal of Neuroscience, 11, 2281-2287.

Osgood, C. E. 1952. The nature and measurement of meaning. Psychological Bulletin, 49, $197-237$.

Posner, M. I., Petersen, S. E., Fox, P. T., \& Raichle, M. E. 1988. Localization of cognitive operations in the brain. Science, 240, 1627-1631.

Rosengren, I. 1977. Ein Frequenzwörterbuch der deutschen Zeitungssprache. Köpenhamn: Villadsen \& Christensen.

Rösler, F., Pütz, P., Friderici, A., \& Hahne, A. 1993. Event-related brain potentials while encountering semantic and syntactic constraint violations. Journal of Cognitive Neuroscience, 5, 345-362.

Samar, V. J., \& Berent, G. P. 1986. The syntactic priming effect: Evoked response evidence for a prelexical locus. Brain and Language, 28, 250-272.

Teyler, T. J., Roemer, R. A., \& Harrison, T. F. 1973. Human scalp-recorded evoked-potential correlates of linguistic stimuli. Bulletin of the Psychonomic Society, 1, 333-334.

Van Petten, C., \& Kutas, M. 1991. Influence of semantic and syntactic context on open and closed-class words. Memory and Cognition, 19, 95-112.

Wackermann, J., Lehmann, D., Michel, C. M., \& Strik, W. K. 1993. Adaptive segmentation of spontaneous EEG map series into spatially defined microstates. International Journal of Psychophysiology, 14, 269-283.

Warrington, E. K., \& McCarthy, R. 1983. Category specific access dysphasia. Brain, 106, 859-878.

Warrington, E. K., \& Shallice, T. 1984. Category specific semantic impairments. Brain, 107, 829-854.

Warrington, E. K., \& McCarthy, R. 1987. Categories of knowledge: Further fractations and an attempted integration. Brain, 110, 1273-1296.

Wetzel, W. W., \& Molfese, D. L. 1992. The processing of presuppositional information contained in sentences: Electrophysiological correlates. Brain and Language, 42, 286-307.

Wise, R., Chollet, F., Hadar, U., Friston, K., Hoffner, E. \& Frackowiak, R. 1991. Distribution of cortical neural networks involved in word comprehension and word retrieval. Brain, 114, 1803-1817.

Zingeser, L. B., \& Berndt, R. S. 1988. Grammatical class and context effects in a case of pure anomia: Implications for models of language production. Cognitive Neuropsychology, $\mathbf{5}$, 473-516.

Zingeser, L. B., \& Berndt, R. S. 1990. Retrieval of nouns and verbs in agrammatism and anomia. Brain and Language, 39, 14-32. 